\title{
COMPARAÇÃO DE METODOLOGIAS DE DETERMINAÇÃO DO COEFICIENTE DE DISPERSÃO PARA O CLORETO DE CÁLCIO EM UM LATOSSOLO VERMELHO-AMARELO
}

\author{
Luiz Fernando Coutinho de Oliveira ${ }^{1}$, Daniel Fonseca de Carvalho $^{2}$ \\ e Paulo Afonso Ferreira ${ }^{3}$
}

\begin{abstract}
RESUMO
Com o objetivo de se comparar os métodos de ajuste gráfico e a regressão não-linear na determinação do coeficiente de dispersão, montaram-se três colunas de solo, através das quais se fez passar uma solução deslocadora de cloreto de cálcio $\left(\mathrm{CaCl}_{2}\right)$, o que permitiu a obtenção dos pontos da curva de eluição. Para efeito de comparação das curvas de eluição ajustadas à experimental, empregaram-se as soluções analíticas das equações diferenciais para a dispersão longitudinal, dispersão para um pulso, difusivo-convectivo e geral do transporte de solutos. Os valores dos coeficientes de dispersão obtidos pelos métodos empregados mostraram que o ajuste gráfico, quando bem empregado, é uma alternativa quando não se dispõe de recursos computacionais para usar o ajuste através de regressão não-linear. As soluções analíticas descrevem com perfeição a curva de eluição, desde que se conheça o coeficiente de dispersão.
\end{abstract}

Palavras-chave: transporte de soluto, dispersão hidrodinâmica, curva de eluição

\section{COMPARISON OF DETERMINATION METHODS OF DISPERSION COEFFICIENT FOR CALCIUM CHLORIDE IN A RED YELLOW LATOSSOLO SOIL}

\begin{abstract}
To compare the graphic adjustment and the non-linear regression methods to obtain points of the breakthrough curve, a solution was passed through three soil columns to displace calcium chloride and provide the elution curve points. To compare the adjusted elution curves with the experimental curve, the differential equations for longitudinal dispersion, dispersion for one pulse, difusiveconvective, and general solute transport analytical solutions were used. The obtained dispersion coefficient values showed that the graphic adjustment, when adaquately applied, is an useful alternative under limited computing resources for adjustment through non-linear regression. The analytical solutions perfectly describe the breakthrongh curve, provided the dispersion coefficient is known.
\end{abstract}

Key words: solute transport, dispersion coefficient, breakthrough curve

\footnotetext{
${ }^{1}$ Engenheiro Agrícola, Professor Assistente da Escola de Agronomia da UFG, CP 131, CEP 74001-970, Goiânia, GO, fax (062) 205- 1099, E.mail: lfco@agro.ufg.br

${ }^{2}$ Engenheiro Agrícola, Professor Assistente da UFRRJ, e.mail:carvalho@ufrrj.br

${ }^{3}$ Engenheiro Agrônomo, Professor Titular da UFV, Bolsista do CNPq, E.mail: pafonso@ mail.ufv.br
} 


\section{INTRODUÇÃO}

O conceito de deslocamento de fluidos miscíveis tem sido empregado em estudos de movimento de solutos no solo. Este conceito pode ser caracterizado, na prática, pelo deslocamento no perfil do solo dos produtos químicos utilizados na agricultura sob a ação combinada do processo de difusão molecular, fluxo de massa e dispersão. As mudanças de concentração do soluto com a posição e com o tempo são explicadas com o auxílio de modelos matemáticos, que resolvem a equação diferencial do transporte de solutos (Equação 1).

$$
\frac{\partial \theta \mathrm{RC}}{\partial \mathrm{t}}=\frac{\partial}{\partial \mathrm{x}}\left[\varepsilon \mathrm{q} \frac{\partial \mathrm{C}}{\partial \mathrm{x}}\right]+\frac{\partial}{\partial \mathrm{x}}\left[\mathrm{D}_{\mathrm{m}} \frac{\partial \mathrm{c}}{\partial \mathrm{x}}\right]-\frac{\partial(\mathrm{qC})}{\partial \mathrm{x}}
$$

em que:

$\theta \quad$ - umidade volumétrica, $\mathrm{L}^{3} \mathrm{~L}^{-3}$

$\mathrm{R}$ - fator de retardamento, adimensional

$\mathrm{C}$ - concentração do soluto na fase líquida do solo, $\mathrm{L} \mathrm{T}^{-3}$

$\varepsilon$ - dispersividade, $\mathrm{L}$

$\mathrm{q}$ - fluxo de água no solo, $\mathrm{L} \mathrm{T}^{-1}$

$\mathrm{D}_{\mathrm{m}}$ - coeficiente de difusão molecular do soluto no solo, $\mathrm{L}^{2} \mathrm{~T}^{-1}$

$\mathrm{t}$ - tempo, $\mathrm{T}$ e

$\mathrm{x}$ - posição da frente de contaminação, $\mathrm{L}$.

A soma das parcelas referentes aos efeitos dispersivodifusivo na equação do transporte de solutos, permite uma simplificação em termos de parâmetros, ou seja:

$$
\frac{\partial \theta R C}{\partial t}=\frac{\partial}{\partial x}\left[D \frac{\partial C}{\partial x}\right]-\frac{\partial(q C)}{\partial x}
$$

D - coeficiente de dispersão hidrodinâmica, $\mathrm{L}^{2} \mathrm{~T}^{-1}$.

Os resultados de experimentos de deslocamento miscível são geralmente interpretados através da análise das mudanças de concentração do soluto no efluente de uma camada de solo, colocados numa forma-padrão chamada curva de efluente. Nielsen \& Biggar (1961 e 1962) e Biggar \& Nielsen (1962 e 1967) verificaram, experimentalmente, que o formato e a posição das curvas de efluente dependem, para um mesmo tipo de solo, do tamanho dos agregados e da magnitude da velocidade convectiva.

As curvas de efluente obtidas experimentalmente podem ser descritas por meio de modelos matemáticos. Com a introdução de modelos mais sofisticados, um importante problema se refere à quantificação dos coeficientes de dispersão, fatores de retardamento e constantes de degradação. Um método popular para a determinação desses parâmetros é o ajuste gráfico aos dados observados, porém de precisão questionável, quando não bem empregado. Em particular, o ajuste por regressão não-linear pelo emprego do método dos quadrados mínimos inverso temse mostrado como excelente ferramenta para esses processos de identificação; portanto, o objetivo deste trabalho consiste na comparação entre metodologias de determinação dos parâmetros da equação de transporte de solutos no solo, com base nos pontos da curva de eluição.

\section{METODOLOGIA}

Para a obtenção dos pontos da curva de eluição, montaramse três colunas de solo de $4,5 \mathrm{~cm}$ de diâmetro e $11 \mathrm{~cm}$ de altura.
O solo utilizado no preenchimento das colunas foi Latossolo Vermelho-Amarelo, variação Una, o qual foi passado em uma peneira de 2,0 mm e seco ao ar. As colunas foram interligadas aos frascos de Mariotte para a manutenção de uma carga hidráulica constante. Inicialmente, promoveu-se a lixiviação da coluna de solo com água destilada, quando foi obtido um valor de condutividade elétrica de $0,00422 \mathrm{dS} \mathrm{m}^{-1}$; em seguida, promoveu-se a contaminação da coluna de solo com uma solução deslocadora de $\mathrm{CaCl}_{2}$ a 0,1 molar; a condutividade elétrica dessa solução $\left(C_{\mathrm{o}}\right)$ foi de $13,46 \mathrm{dS} \mathrm{m}^{-1}$.

A solução eluente foi coletada em intervalos de tempo suficiente para se recolher amostras a cada 0,2 volume de poros, até totalizar dois volumes. A concentração da solução eluente foi determinada indiretamente pelo emprego de um condutivímetro. Com base nos pontos da curva de eluição, determinaram-se os coeficientes de dispersão empregando-se os métodos de ajuste gráfico e por regressão não-linear.

Para efeito de comparação aos dados observados, empregaram-se as soluções analíticas desenvolvidas por Kirkham \& Powers (1972) para a solução das equações diferenciais para a dispersão longitudinal, dispersão para um pulso e difusão e fluxo de massa e a desenvolvida por Parker \& van Genucthen (1984) para a solução da equação geral do transporte de solutos, conforme segue:

\section{Modelo 1: Solução analítica para a dispersão longitudinal}

$$
\begin{gathered}
C(x, t)=C_{i}+\frac{\left(C_{o}-C_{i}\right)}{2}\left[1-\operatorname{erf}\left(\frac{1-p}{\sqrt{4 D p / v x}}\right)\right] \\
D=\frac{v L_{c s}}{4 \pi S^{2}}
\end{gathered}
$$

em que:

$\mathrm{C}(\mathrm{x}, \mathrm{t})$ - concentração do soluto na fase líquida do solo na posição x e tempo t, $\mathrm{L} \mathrm{T}^{-3}$,

$\mathrm{C}_{\mathrm{i}}$ - concentração inicial da solução do solo, $\mathrm{L} \mathrm{T}^{-3}$

$\mathrm{C}_{\mathrm{o}}^{\mathrm{i}} \quad$ - concentração da solução deslocadora, $\mathrm{L} \mathrm{T}^{-3}$

$\mathrm{D}^{\mathrm{o}}$ - coeficiente de dispersão, $\mathrm{L}^{2} \mathrm{~T}^{-1}$

v - velocidade média da solução no solo, $\mathrm{L} \mathrm{T}^{-1}$

p - número de volume de poros

$\mathrm{L}_{\mathrm{cs}} \quad$ - comprimento da coluna de solo, $\mathrm{L}$

$\mathrm{S}^{\mathrm{cs}} \quad$ - declividade da curva experimental para $\mathrm{p}=1$

erf - função - erro obtida por erf $=1$ - erfc, sendo erfc $=$ função-erro complementar.

Modelo 2: Solução analítica para a dispersão de um pulso

$$
\frac{\mathrm{C}}{\mathrm{Co}}=\frac{1}{2}\left\{\left[\operatorname{erf} \frac{1+\frac{\mathrm{x}_{\mathrm{o}}}{\mathrm{L}}-\mathrm{p}}{2[\mathrm{Ep} /(\mathrm{vL})]^{1 / 2}}\right]-\left[\operatorname{erf} \frac{1-\mathrm{p}}{2[\mathrm{E} \mathrm{p} /(\mathrm{v} \mathrm{L})]^{1 / 2}}\right]\right\}
$$

em que:

$\mathrm{x}_{\mathrm{o}}-\mathrm{Vs} / \alpha \mathrm{A}$

Vs - volume da solução, $\mathrm{L}^{3}$

A - área transversal de escoamento, $\mathrm{L}^{2}$

$\mathrm{p}-1+\mathrm{x}_{\mathrm{o}} / 2 \mathrm{~L}_{\mathrm{cs}}$

D - coeficiente de dispersão, $\mathrm{L}^{2} \mathrm{~T}^{-1}$, obtido pela Eq. (6).

$$
\mathrm{D}=\frac{\mathrm{vL}_{\mathrm{cs}}\left(\mathrm{x}_{\mathrm{o}} / 2 \mathrm{~L}_{\mathrm{cs}}\right)^{2}}{2\left(1+\mathrm{x}_{\mathrm{o}} / 2 \mathrm{~L}_{\mathrm{cs}}\right) \mathrm{Z}^{2}}
$$


em que:

$\mathrm{Z}$ - coordenada da distribuição normal em função da relação $0,5 \mathrm{C} / \mathrm{C}_{0}$ máxima na curva de eluição obtida experimentalmente, sendo $\mathrm{C}$ - concentração da solução eluente e $\mathrm{C}_{\mathrm{O}}$ - concentração da solução deslocadora.

Modelo 3: Solução analítica para difusão e fluxo de massa

$$
\left.\frac{\mathrm{C}}{\mathrm{Co}}=\frac{1}{2}\left\{\left[\operatorname{erfc} \frac{1-\mathrm{p}}{2[\mathrm{D} \mathrm{p} /(\mathrm{vL})]^{1 / 2}}\right]+\mathrm{e}^{\frac{\mathrm{vL}}{\mathrm{D}}} \operatorname{erfc} \frac{1+\mathrm{p}}{2[\mathrm{D} \mathrm{p} /(\mathrm{vL})]^{1 / 2}}\right]\right\}
$$

em que:

D - coeficiente de difusão, $\mathrm{L}^{2} \mathrm{~T}^{-1}$, obtido pela Equação (4).

Modelo 4: Solução analítica para a equação geral do transporte de solutos, considerando-se $R=1$

$$
\begin{gathered}
C(x, t)=C_{i}+\left(C_{o}-C_{i}\right) A(x, t)+B(x, t) \\
A(x, t)=\frac{1}{2} \operatorname{erfc}\left(\frac{x-v t}{\sqrt{4 D t}}\right)+\frac{1}{2} \exp \left(\frac{v x}{D}\right) \operatorname{erfc}\left(\frac{x+v t}{\sqrt{4 D t}}\right) \\
B(x, t)=t+\left(\frac{x-v t}{2 v}\right) \operatorname{erfc}\left(\frac{x-v t}{\sqrt{4 D t}}\right)-\left(\frac{x+v t}{2 v}\right) \exp \left(\frac{v x}{D}\right) \operatorname{erfc}\left(\frac{x+v t}{\sqrt{4 D t}}\right)
\end{gathered}
$$

Modelo 5: Solução analítica para a equação geral do transporte de solutos

$$
\begin{gathered}
C(x, t)=C_{i}+\left(C_{o}-C_{i}\right) A(x, t)+B(x, t) \\
A(x, t)=\frac{1}{2} \operatorname{erfc}\left(\frac{R x-v t}{\sqrt{4 D R t}}\right)+\frac{1}{2} \exp \left(\frac{v x}{D}\right) \operatorname{erfc}\left(\frac{R x+v t}{\sqrt{4 D R t}}\right) \\
B(x, t)=\frac{1}{R}\left[t+\left(\frac{R x-v t}{2 v}\right) \operatorname{erfc}\left(\frac{R x-v t}{\sqrt{4 D R t}}\right)-\left(\frac{R x+v t}{2 v}\right) \exp \left(\frac{v x}{D}\right) \operatorname{rrf}\left(\frac{R x+v t}{\sqrt{4 D R t}}\right)\right]
\end{gathered}
$$

A função erfc foi obtida pela aproximação proposta por Abramowitz \& Stegun (1970) apud Beljin (1985):

$$
\operatorname{erfc}(x)=\frac{}{\left[1+a_{1} x+a_{2} x^{2}+\ldots+a_{6} x^{6}\right]^{16}}+E(x)
$$

em que:

$$
\begin{aligned}
\mathrm{E}(\mathrm{x}) & =\text { erro de truncamento, }|\mathrm{E}(\mathrm{x})| \leq 3 \times 10^{-7} \\
\mathrm{a}_{1} & =0,0705230784 \\
\mathrm{a}_{2} & =0,0422820123 \\
\mathrm{a}_{3} & =0,0092705272 \\
\mathrm{a}_{4} & =0,0001520143 \\
\mathrm{a}_{5} & =0,0002765672 \\
\mathrm{a}_{6} & =0,0000430638
\end{aligned}
$$

As soluções analíticas anteriormente apresentadas permitiram o traçado da curva de eluição para os parâmetros ajustados pelos dois métodos utilizados neste trabalho. $\mathrm{O}$ ajuste das curvas de eluição obtidas via solução analítica aos dados observados, foi avaliado pelo emprego do erro relativo médio (ERM) e do desvio-padrão (DP) segundo Alexander \& Skaggs (1986 e 1987) conforme segue:

$$
\text { ERM }=\frac{\sum_{\mathrm{i}=1}^{\mathrm{n}}\left|\frac{\mathrm{Y}_{\mathrm{oi}}-\mathrm{Y}_{\mathrm{ei}}}{\mathrm{Y}_{\mathrm{oi}}}\right|}{\mathrm{n}}
$$

$$
\mathrm{DP}=\frac{\sqrt{\sum_{\mathrm{i}=1}^{\mathrm{n}}\left|\frac{\mathrm{Y}_{\mathrm{oi}}-\mathrm{Y}_{\mathrm{ei}}}{\mathrm{Y}_{\mathrm{oi}}}\right|^{2}}}{\mathrm{n}}
$$

em que:

$\mathrm{Y}_{\mathrm{O}}$ e $\mathrm{Y}_{\mathrm{e}}$ - pontos da curva de eluição observados e estimados pelas soluções analíticas, respectivamente

n - 11 (número de pontos observados da curva de eluição).

\section{RESULTADOS E DISCUSSÃO}

$\mathrm{O}$ volume de poros para a obtenção dos pontos da curva de efluente $(103,89 \mathrm{~mL})$ foi obtido com base no volume da amostra $(174,9 \mathrm{~mL})$, nas massas específicas do solo e de partículas (1076 e $2650 \mathrm{~kg} \mathrm{~m}^{-3}$, respectivamente) e na porosidade total $(59,39 \%)$.

A Tabela 1 apresenta os pontos experimentais da curva de eluição $\left(\mathrm{C} / \mathrm{C}_{\mathrm{O}}\right.$ versus $\left.\mathrm{p}\right)$. Com base nos pontos experimentais da curva de eluição, obteve-se a declividade para o ponto de inflexão da curva $S=1,1805$, empregado nos modelos 1 e 3 , e a coordenada da distribuição normal $\mathrm{Z}=2,435$ para $0,5\left(\mathrm{C} / \mathrm{C}_{\mathrm{O}}\right)$ $=0,4926$, para o modelo 2. Para os modelos 4 e 5, o coeficiente de dispersão e o fator de retardamento foram obtidos pelo ajuste através da aplicação da regressão não-linear pelo emprego do software CXTFIT desenvolvido por Parker \& van Genucthen (1984).

Tabela 1. Pontos experimentais da curva de eluição do $\mathrm{CaCl}_{2}$ para o Latossolo Vermelho-Amarelo

\begin{tabular}{cccc}
\hline Tempo (segundos) & Volume de Poros & $\begin{array}{c}\mathrm{CE} \\
\left.(\mathrm{dS} \mathrm{m})^{-1}\right)\end{array}$ & $\mathrm{C} / \mathrm{C}_{\mathrm{o}}$ \\
\hline- & 0,0 & 0,296 & 0,0220 \\
540 & 0,2 & 0,186 & 0,1380 \\
1080 & 0,4 & 0,129 & 0,0096 \\
1670 & 0,6 & 0,368 & 0,0273 \\
2110 & 0,8 & 3,290 & 0,2440 \\
2820 & 1,0 & 6,000 & 0,4458 \\
3340 & 1,2 & 8,740 & 0,6493 \\
3900 & 1,4 & 11,420 & 0,8484 \\
4500 & 1,6 & 11,810 & 0,9391 \\
5040 & 1,8 & 12,000 & 0,9539 \\
5640 & 2,0 & 13,260 & 0,9851 \\
\hline
\end{tabular}

Com base nos dados do ensaio e nas características geométrias da coluna e do solo, obteveram-se os valores do fluxo de água no solo $\left(\mathrm{q}=2,3,10^{-5} \mathrm{~m} \mathrm{~s}^{-1}\right)$ e da velocidade convectiva $\left(\mathrm{v}=3,910^{-5} \mathrm{~m} \mathrm{~s}^{-1}\right)$ utilizados para a solução de todos os modelos apresentados.

A Figura 1 mostra os pontos observados e as curvas de eluição obtidas pelas soluções analíticas. Observa-se, na referida figura, um perfeito ajuste para os métodos de obtenção do coeficiente de dispersão e para as diferentes soluções analíticas.

A Tabela 2 apresenta os valores dos coeficientes de dispersão (D) ajustados pelos métodos gráficos e pela regressão não-linear, os erros relativos médios e desvio padão entre os dados observados e ajustados. Os valores obtidos para os erros relativos médios (ERM) e desvio padrão (DP) para os cinco modelos analisados foram relativamente baixos, o que mostra um bom ajuste aos dados observados, evidenciando as curvas de eluição 


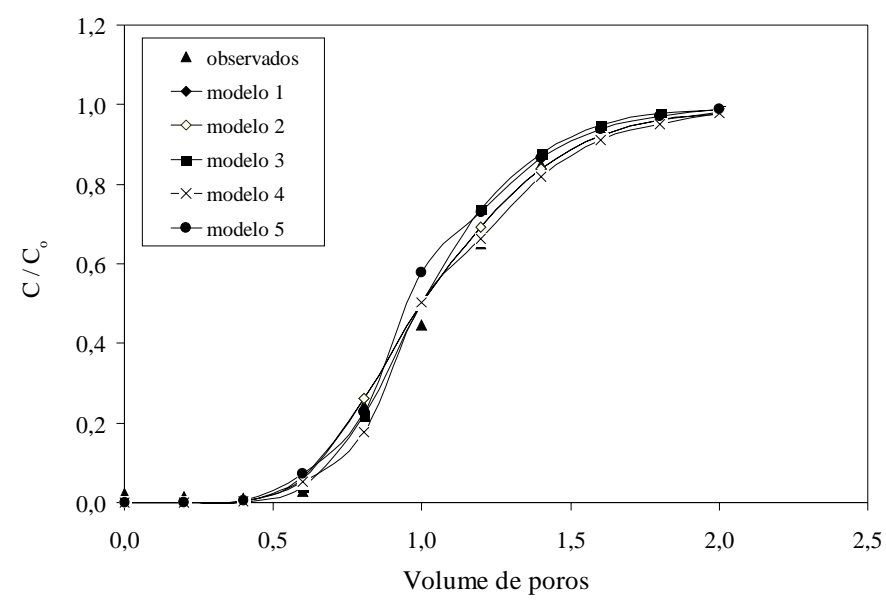

Figura 1. Curvas de eluição obtidas pelas soluções analíticas

Tabela 2. Valores de coeficientes de dispersão (P), fator de retardamento $(\mathrm{R})$, erros relativos médios (ERM) e desviopadrão (DP) para os modelos estudados

\begin{tabular}{ccccc}
\hline Modelo & $\begin{array}{c}\mathrm{D} \\
\left(\mathrm{M}^{2} \mathrm{~S}^{-1}\right)\end{array}$ & $\mathrm{R}$ & $\mathrm{ERM}$ & $\mathrm{DP}$ \\
\hline 1 & $2,44 \mathrm{E}-07$ & - & 0,3983 & 0,0079 \\
2 & $1,81 \mathrm{E}-07$ & - & 0,3450 & 0,0107 \\
3 & $2,45 \mathrm{E}-07$ & - & 0,3871 & 0,0078 \\
4 & $2,52 \mathrm{E}-07$ & 1,000 & 0,3772 & 0,0095 \\
5 & $2,59 \mathrm{E}-07$ & 1,067 & 0,4344 & 0,0151 \\
\hline
\end{tabular}

apresentadas na Figura 1; portanto, o ajuste gráfico, quando bem empregado representa uma alternativa, quando não se dispõe de recursos computacionais, para se usar o ajuste através de regressão não-linear. $\mathrm{O}$ valor de $\mathrm{D}$ obtido para o Modelo 2 foi menor que os demais, pelo fato de se considerar que a curva de eluição para aplicação de um pulso da solução deslocadora tem a forma da curva normal. $\mathrm{O}$ fator de retardamento ajustado para o Modelo 5 ficou próximo de um, o que mostra que o íon cloreto não é adsorvido pela fração argila do solo.

\section{CONCLUSÕES}

Com base nos resultados obtidos, pode-se concluir que o método gráfico, quando bem empregado, representa uma alternativa na obtenção do coeficiente de dispersão e que as soluções analíticas descrevem com perfeição a curva de eluição, desde que se conheça o coeficiente de dispersão.

\section{REFERÊNCIAS BIBLIOGRÁFICAS}

ALEXANDER, L.; SKAGGS, R.W. Predicting unsaturated hydraulic conductivity from the soil water characteristics. Transactions of the ASAE, St. Joseph, v.23, n.2, p.176184, 1986.

ALEXANDER, L.; SKAGGS, R.W. Predicting unsaturated hydraulic conductivity from the soil texture. Journal of Irrigation and Drainage Enginnering, New York, v.113, n.2, p.184-197, 1987.

BELJIN, M.S. A program package of analytical models for solute transport in groundwater "solute". Indianapolis: Holcomb Research Institute, 1985, 163 p.

BIGGAR, J.M.; NIELSEN, D.R. Miscible displacement: II, Behavior of tracers. Soil Science Society of America Proceedings, Madison, v.26, n.2, p.125-128, 1962.

BIGGAR, J.M.; NIELSEN, D.R. Miscible displacement and leaching phenomenon. In: HIGAN, H.R.; EDMINSTER, T.W. ed. Irrigation of Agricultural lands, Madison, American Society of Agronomy, 1967, p.254-274.

KIRKHAM, D.; POWERS, W.L. Advanced Soil Physics. New York: Wiley-Interscience, 1972. 534 p.

NIELSEN, D.R.; BIGGAR, J.W. Miscible displacement: I, Experimental information. Soil Science Society of America Proceedings, Madison, v.26, n.1, p.1-5, 1961.

NIELSEN, D.R.; BIGGAR, J.W. Miscible displacement: III, Theoretical considerations. Soil Science Society of America Proceedings, Madison, v.26, n.3, p.216-221, 1962.

PARKER, J.C., van GENUCHTEN M.Th. Determining transport parameters from laboratory and field tracer experiments. Virginia-EUA: Virginia Agricultural Experiment Station, 1984. 96p. Bulletin, 84-3 